\title{
Historia crítica de la Psiquiatría en el siglo XX. Una mirada biográfica
}

El tema de mi conferencia es una mirada biográfica, es decir, una mirada de testigo sobre la psiquiatría que me fue dado encontrar en mi etapa de formación. Hace 63 años que penetré en el universo psiquiátrico para no volver a salir de él. Yo tenía veinte años y estudiaba $4 .^{\circ}$ año de Medicina.

En 1934, cuando tenía 12 años, mi mentor, de la Institución Libre de Enseñanza, que idealizaba a D. Santiago Ramón y Cajal -que moriría el 17 de Octubre de ese mismo año- y me hablaba de su ejemplo, puso en mis manos su autobiografía, Recuerdos de mi Vida (que se acaba de reeditar por la editorial «Crítica» como obra del canon científico del siglo XX). Por entonces tenía yo un laboratorio de Histología en mi casa y gracias a este mentor y al médico de mi pueblo penetré en ese universo. En aquella época los médicos generales leían mucho y el mío tenía los diecisiete volúmenes de la primera traducción de la obra de Freud realizada por López Ballesteros, que me fue dejando uno tras otro.

Si bien es cierto que a mí me interesan muchas cosas, demasiadas quizás para lo que yo puedo abarcar, cabe decir que mi mundo, mi universo, ha sido el universo psiquiátrico, o para ser más exacto, el psico(pato)lógico, que incluye al psiquiátrico pero que contiene muchos más. Todo lo demás han sido, por decirlo así, escarceos, excursiones, paseos. Algo parecido le ocurría a Nabokov: cazaba mariposas, las estudiaba y describía, e incluso algunas veces añadía alguna especie, género o familia a las ya clasificadas por los lepidopterólogos. Pero, sobre todo, escribía, porque Nabokov, antes que cualquier otra cosa, era escritor. Y yo, salvando todas las diferencias con Nabokov, soy ante todo psiquiatra.

\section{La Psiquiatría en el Madrid de 1940}

La psiquiatría que me encontré en 1942, cuando comencé mi formación en el Servicio de Psiquiatría del Hospital General de Madrid, era en realidad de Neuropsiquiatría, es decir que era de Neurología y de Psiquiatría. Entonces se era neurólogo y psiquiatra porque, salvo en Cataluña, que se seguía el modelo francés, que ya desde el siglo XIX había escindido la Neurología y la Psiquiatría, en el resto de España el modelo adoptado era el alemán (incluso el norteamericano) que las mantenía unidas. No hay que olvidar el número de revistas que por entonces se llamaban de Neurología y de Psiquiatría, por ejemplo: los Archives de 
Neurology and Psychiatry o los Excerpts de Neurology and Psychiatry, Fortschrite der Neurologie und Psychiatry, etc. Luego, en la práctica, cada cual se inclinaba más hacia la Neurología o hacia la Psiquiatría.

Éste era el caso de López Ibor, Jefe del Servicio de Neuropsiquiatría del Hospital General, hoy Museo «Reina Sofía». López Ibor era, por así decirlo, más psiquiatra que neurólogo, pero sabía bastante de Neurología porque había opositado a una plaza de Neurología en el Hospital Provincial de Valencia, que, aunque no obtuvo, le sirvió para adquirir conocimientos en ese campo. Al ocupar el Servicio de Neuropsiquiatría del Hospital General de Madrid, recibió la influencia de uno de los más grandes neurólogos españoles, desgraciadamente muerto cuando tenía tan solo 39 años, que ocupó la dirección del Servicio de Psiquiatría de Leganés: Manuel Peraita. A Peraita le ocurría lo contrario que a López Ibor, que era más neurólogo que psiquiatra. Se había formado en Alemania con Förster y Altenburger y la Psiquiatría le venía, por así decirlo, por añadidura. En Madrid, todo psiquiatra que se preciase de tal debía de saber Neurología, y todo neurólogo que se preciase de tal debía de saber Psiquiatría y, a ser posible, también Neuropatología, sobre todo si se tenían las pretensiones, como las tenía yo, de acceder con cierto decoro a una cátedra universitaria.

Con respecto a la Neuropatología, hay que tener en cuenta algunos matices de importancia en la historia para la Psiquiatría española del siglo XX y de lo que llevamos del XXI. En España, Cajal no hizo nunca neuropatología, y sólo publicó un trabajo acerca de las alteraciones de la neuroglía en la parálisis general sifilítica (PGP). Cajal era un neurohistólogo de un talento y una habilidad técnica asombrosos. Cajal pensaba que había un hueco en la escuela española, el de la Neuropatología. Había importantes neurohistólogos como D. Fernando de Castro, D. Domingo Sánchez (que trabajó con Cajal y escribieron juntos una monografía sobre la retina de los insectos), Tello, Calandre, Lafora, Ramón y Fañanás, etc. La escuela española tenía relevancia, además, por la habilidad en la creación de métodos de tinción, pero, repito, no había desarrollado la Neuropatología. Por fortuna para Cajal, apareció en España Nicolás Achúcarro, discípulo de Nissl, Alzheimer y Kraepelin, que ocupó el puesto de Jefe del Servicio de Neuropsiquiatría, el mismo en el que yo trabajaría, y que ocuparía más tarde, andando los años, Villaverde (asesinado en Madrid en 1936) en la sala de hombres y Lafora (exiliado en México) el de mujeres, y tras la guerra civil, López Ibor. Por desgracia, Achúcarro murió muy joven de la enfermedad de Hodking. Todo el que le trató reconoció que era una personalidad verdaderamente genial, y alguien, nada sospechoso de beatería, como Ortega y Gasset, que le hizo una necrológica, reconoció que Achúcarro era una de las personas de más talento que había conocido en su vida. 
Era frecuente que el psiquiatra que pretendía adquirir una formación y prepararse para el magisterio universitario pasase por el laboratorio de Neurohistología. Un psiquiatra en sentido estricto como José Miguel Sacristán, maestro de tantas generaciones, escribió un trabajo sobre la histología de la epífisis, importante para su época. De manera que el paso por el laboratorio era, por lo menos para mí, que seguía los pasos de lo que sabía que era la tradición, muy necesario, aunque los psiquiatras de la época, como el propio López Ibor y no hablemos Sarró, no estaban en esa línea, aunque sí otro, el malogrado Román Alberca, que hizo neuropatología en París en sus años primeros.

\section{La nosología}

Por entonces, la nosología que utilizábamos en el Servicio de Neuropsiquiatría era la de Kraepelin con algunas modificaciones como, por ejemplo, las introducidas por Kretschmer (el delirio sensitivo de referencia, independiente de cualquier proceso esquizofrénico, era una aportación de Kretschmer). También se recogían algunas conceptualizaciones de la nosología de la escuela de Karl Kleist, el profesor de Frankfurt, y su discípulo Leonhard, una nosología en muchos aspectos muy arbitraria y de una tendencia localizacionista exagerada. Toda la bibliografía que manejábamos en Madrid era alemana. La Psiquiatría francesa se soslayó hasta que, unos años después, poco antes del Congreso de Neuropsiquiatría de Zurich, de 1956, penetró en España a través de Delay, Henry Ey, Giraud, Baruk, Pichot, etc. De Baruck se conocía el tratado de psiquiatría biológica que había publicado en los años treinta. Yo poseo un ejemplar que perteneció al psiquiatra Anguera de Sojo, vendido, entre otros muchos, por su familia cuando fue represaliado después de la guerra civil.

\section{Los textos}

Los libros de psiquiatría en aquella época más en uso eran los siguientes: la última edición del Tratado de las Enfermedades Mentales, de Oswald Bunke; la espléndida Psiquiatría de Martin Reichard, cuyos capítulos estaban escritos por distintos psiquiatras alemanes y suizos; el Tratado de Psiquiatría de Eugen y Manfred Bleuler; la Psiquiatría de Johannes Lange, continuada por su discípulo Bostroem. Lange fue el último discípulo de Kraepelin y había de sucederle en la dirección del Instituto de Investigaciones Psiquiátricas de Munich. La introducción psicopatológica del libro de Lange-Bostroem es realmente extraordinaria, y 
aún ahora se puede leer con sumo provecho por su finura de análisis. Hoy que tanto se discute la teoría del síntoma psiquiátrico, se podría aprender bastante de esta psicopatología de Lange-Bostroem.

En cuanto a la psicopatología, utilizábamos la obra de Jaspers, pero no en su edición alemana (que estaba agotada), sino en su edición francesa. La quinta edición alemana, modificada ya un tanto por Kurt Schneider, apareció en 1945 y, naturalmente, algunos la adquirimos para devorarla. Jaspers, aunque abandonó la psiquiatría muchos años antes, dejó su influjo en lo que se denominó la escuela de Heidelberg, que la dirigía Willmans con Gruhle, Mayer-Gross, Karl y Kurt Schneider. Bumke, que dirigió el tomo undécimo de Handbuch Der Geisteskrankheiten dedicado totalmente a la esquizofrenia, hizo que lo dirigiera Willmans y sus colaboradores.

\section{Neuropsiquiatría}

La unidad «Neuropsiquiatría» tenía una tradición histórica que se inicia en Alemania con Griesinger, Meiner, Gudden y Wernicke, entre otros. Hay que tener en cuenta que el concepto de disgregación esquizofrénica que manejamos en la actualidad fue descrito por Wernicke, que le llamó «sejunción». Karl Wernicke era un neurólogo que hizo aportaciones sobre las afasias de carácter absolutamente indelebles.

Desde el punto de vista histórico, una de las razones para que la Neurología y la Psiquiatría no se separasen era la existencia de la Parálisis General Progresiva (PGP). Entre una polineuritis y una esquizofrenia no hay posibilidad de contacto, pero ¿qué se hace cuando nos encontramos con una PGP?, ¿es de la Neurología o de la Psiquiatría?; o con un caso de Alzheimer: ¿es de una y no de la otra? Yo creo que esta discusión no tiene sentido. Era necesario adquirir una formación con un nuevo carácter, que no tiene que ser necesariamente de Neurología en el sentido más amplio, pero sí de patología cerebral en un sentido restringido.

Como digo, había una enfermedad que nos obligaba al mantenimiento de este concepto de Neuropsiquiatría que era la PGP. Nosotros teníamos en el servicio sangre de palúdico, que guardábamos con oxalato para que no se coagulara y para inyectársela al próximo paralítico general que apareciera, porque teníamos en la sala de hombres entre 8 y 14 ó 15 paralíticos ingresados (en la de mujeres menos), en los cuales producíamos accesos febriles. En el año 1946, cuando la penicilina adquiere toda su vigencia y su carta de naturaleza en Medicina, desaparece la PGP que nos había enseñado tanto sobre la patología del lóbulo prefrontal, ya que los tumores frontales eran relativamente infrecuen- 
tes. La patología del lóbulo frontal, la llamada «moria», que describió Jastrovich como síndrome del mismo a finales del XIX, la encontrábamos en los PGP, a veces como síntoma de comienzo.

Recuerdo el caso de un famoso odontólogo que había en Madrid que fue diagnosticado de PGP y que comenzó a suscitar extrañeza por sus extravagancias. En una ocasión, cuando un paciente tenía puestos los artilugios y la boca abierta, él le dijo: «Permítame por unos momentos que descanse», y a continuación se echó allí mismo en un diván. Otra vez se arrancó un diente sano, porque «así puedo escupir sin abrir la boca». Conductas todas que correspondían al síndrome prefrontal. Pero, claro está, en la exploración neurológica estos enfermos mostraban, además, el síndrome pupilar de Argill-Robertson, la disartria, la exaltación de patelares, la fibrilación lingual, etc.

Yo invitaría a todos aquellos que estén interesados en la historia de la Psiquiatría a leer los primeros libros de Kraepelin y los de un contemporáneo suyo que, aunque no fuera tan relevante como Kraepelin, no eran menos interesantes: me refiero a $\mathrm{H}$. Schüle, cuyo tratado es de 1888. En muchas de sus páginas se dedican a los malabarismos clínicopsicológicos que han de hacerse para diferenciar en los comienzos lo que luego podía ser una demencia paralítica (PGP) o una depresión del adulto, con síntomas de lesión cerebral sobreañadidos. Esas depresiones del adulto, que entonces eran manicomiables, tenían en su mayoría una, por decirlo así, impronta orgánica, lo que hoy se ha vuelto a resucitar como síndrome seudodemencial en la depresión. Esta perspicacia clínica era obligada, porque aún no había posibilidad de hacer una serología que aclarase la etiología sifilítica de la PGP, y Noguchi no describiría hasta 1908 la presencia del treponema pallidum en el córtex cerebral de los paralíticos generales. Hasta esa fecha, pues, había que valerse exclusivamente de la clínica.

\section{La patología cerebral, frontera neuropsiquiátrica}

Neurología y Psiquiatría tenían una línea fronteriza. Era el capítulo de las afasias, apraxias y agnosias. La patología cerebral propiamente dicha se calificaba precisamente como ese Grenzegebiete, como dicen los alemanes, «territorio límite», entre la Neurología y la Psiquiatría. Hay que recordar que el propio Freud, además de neurohistólogo en sus comienzos, cuando se hace clínico es en realidad neuropsiquiatra, antes de llegar a ser el creador del psicoanálisis. Recuerden sus trabajos sobre encefaloneuropatías infantiles y su aportación a la doctrina de las afasias, que todavía se puede leer con gran provecho. La doctrina de las afasias, agnosias y apraxias resolvía, además, el problema de la relación entre las estructuras cerebrales y el aprendizaje, y era el principal argumento a favor del organicismo. Todavía hoy, espe- 
cialmente los que son organicistas estrictos y pretenden que la psicopatología sea sustituida por una neurología de imagen, hay quien cree que cuando hablamos de Psicopatología estamos hablando de la mente en términos medievales, como sinónimo de alma, como una entidad de naturaleza inmaterial o algo por el estilo. Hablamos de que los síntomas mentales son alteraciones funcionales corticales que tienen su categoría, y que en lugar de expresarse como una alteración de los reflejos musculares profundos o de la sensibilidad se expresan como alteración mental. Pero con una peculiaridad, a saber, que esta alteración mental afecta a lo individual propiamente dicho. Cuando hablamos de la mente hacemos referencia a estructuras creadas al tiempo que se crea la biografía de cada cual. La biografía de cada uno de nosotros está en nuestro cerebro, y por eso nuestro cerebro es tan irrepetible como lo es nuestra propia vida... Recuerdo que en aquellos años de mi formación, muy joven aún, presenté en una sesión clínica del Servicio de Psiquiatría un caso de síndrome de Gertsmann. Este síndrome se caracteriza por agrafia (pérdida de la capacidad para la escritura) y agnosia para el reconocimiento de los dedos (Fingeragnosie). En la discusión dije que el síndrome de Gertsmann no se podía encontrar entre nosotros en la misma proporción que en Austria, porque el 70\% de la población española no sabía leer ni escribir. Alguien puede tener síndrome de Gertsmann si y sólo si en su cerebro se ha acuñado por aprendizaje la lectura y la escritura, y éste es un rasgo biográfico en sentido estricto. Como ocurre en algunos afásicos que han sido bilingües. Tuve un caso de un bilingüe catalán, que había aprendido el español como segunda lengua, que al tener una afasia perdió el uso del español.

La patología cerebral es una alteración biográfica, como lo es la aparición de una psicosis esquizofrénica. Otro tanto ocurre en la temática de los delirios. Heinrich Kranz, profesor en Mainz, y que pasó por Córdoba, me envió un trabajo en el cual recoge la historia de los temas del delirio desde 1880 hasta 1938-1940. Los temas del delirio van cambiando. Hoy en día es raro que alguien que tenga delirio de grandeza se crea Napoleón, pero en los años treinta se podía creer Hitler. Napoleón no está ya en la hábeas biográfica de la mayoría de la población occidental y es a eso a lo que se debe el que no aparezca como tema de un delirio. Las mismas estructuras del delirio cambian también. Mi propia tesis doctoral trató de un caso de agnosia óptica de una enferma que se llamaba María Madrid, una muchacha de 27años que en el curso de un parto tuvo una embolia que afectó al gyrus angularis y presentaba un trastorno muy curioso: una agnosia para la percepción del movimiento, de manera que era incapaz de reconocer que un objeto se movía en su campo visual... Les digo todo esto para aclarar que la patología cerebral era ese territorio frontera entre la Neurología.

El mismo López Ibor proyectó por entonces un libro para el cual contaba con Xavier Zubiri y conmigo. Zubiri, el filósofo, se interesaba mucho por el problema de la formalización de las estructuras mentales y por el tema de las locali- 
HISTORIA DE LA PSIQUIATRÍA

zaciones cerebrales. Creía que el cerebro era un órgano que constantemente formalizaba la realidad que se percibía, para así conocerla y dominarla. López Ibor iba a tratar sobre las afasias y yo sobre las agnosias. Ese grupo nuestro que tenía inquietudes del tipo de las que le acabo de describir, estaba muy influido por neurólogos muy sutiles, como por ejemplo Kurt Goldstein. Tengan en cuenta que lo que ahora se conoce como síntomas negativos de la esquizofrenia de Andreasen, es lo que Goldstein denominó «pérdida de la capacidad categorial», y por eso puedo afirmar que el primero que describió los síntomas negativos de la esquizofrenia fue Goldstein, un neurólogo que trabajó en el campo de la patología cerebral con los heridos de la Primera Guerra Mundial. El test de Kasanin que utilizábamos en la esquizofrenia se basa en las tesis de Goldstein y describe el descenso de la capacidad de abstracción que tiene lugar en el deterioro esquizofrénico, que es una demencia muy distinta de la demencia tipo Alzheimer, que es esencialmente mnéstica, o la de la PGP, que es netamente frontal. Hay que recordar que la investigación biológica en psiquiatría, que hoy tiene una vigencia tan extraordinaria y ha coronado tantos éxitos, comienza en la década de los años treinta. Recuerden que los primeros hallazgos fisiopatológicos sobre la esquizofrenia fueron los de Gjessing y colaboradores, en Munich, sobre la perturbación del metabolismo proteico en la catatonía. Se trataba de una investigación de laboratorio, muy primitiva en comparación con los actuales. Yo mismo estuve estudiando la histopatología de la catatonía durante mis años de permanencia en el Instituto Cajal, a donde iba por las tardes, porque en España seguía estando vigente un comentario de Harry Stack Sullivan, quien solía recordar que durante sus estudios de psiquiatría en Alemania le advirtieron que no dejara de pasar por España si quería ver catatónicos, infrecuentes en Alemania. Aunque hoy la esquizofrenia catatónica es una rareza, en aquella época teníamos bastantes ingresos de esquizofrenias catatónicas y, muchos de ellos, de la forma llamada «aguda mortal»o «catatonía febril mortal». Yo estudiaba los cerebros de nuestras necropsias en el Instituto Cajal.

\section{El grupo de Madrid en los años cuarenta}

Mi vida de aquellos años fue una conjunción muy afortunada, porque durante algún tiempo era el único estudiante en aquel servicio donde estaban Manuel Peraita y López Ibor, Eugenio Olivares y Bartolomé Llopis, y algunos más. López Ibor conocía bien la psicopatología fenomenológica. Había leído a Brentano y a Husserl. Poco después llegó Lafora, que era fundamentalmente un gran neuropatólogo, y lo digo con el mayor respeto hacia su figura. El hecho de que a los 25 años descubriera la neuropatología de la epilepsia mioclónica de Unverricht, le catapultó desde el 
primer momento entre las autoridades de la Neuropatología mundial de la época. La biblioteca de Lafora contiene trabajos que le están dedicados por las autoridades de la época. He visto separatas de Pavlov y Sherrington dedicadas a Lafora.

Estaba también Llopis, que escribió un libro desafortunadamente poco leído hoy en día, La psicosis pelagrosa. No era este libro el lugar adecuado para hacer una introducción a la psicopatología. Llopis era un psicopatólogo excepcional, y les recomiendo que lean sus 40 ó 50 páginas de psicopatología, sobre todo lo referente a la percepción y las ideas delirantes, que son de una originalidad y claridad sorprendentes. Otra figura importante para mí fue la de Eugenio Olivares, discípulo de Sacristán, que, además de un neuropsiquiatra eminente, era una persona muy culta. Olivares es el que traduce del alemán al español el primer libro de Psiquiatría de la infancia que se edita en nuestro país. Y como neurólogo, Manuel Peraita, del que antes hablé, y que describió la neurología de la pelagra en una monografía espléndida. Yo era el único estudiante. Cuatro años después llegarían Morales, Letemendía, Martín Santos, y algo más tarde Rallo Romero y López de Lerma, y algunos más que se malograron o se aburrieron.

En aquellos años la psicopatología de Jaspers fue sustituida por la de Kurt Schneider, más ligada al diagnóstico. Lo fundamental era la distinción entre forma y contenido: las alteraciones formales se atribuían a las que produce el proceso patológico, por ejemplo, la disgregación o las alucinaciones del esquizofrénico; mientras las del contenido se referían al tema de la alucinación o del delirio o de la obsesión o la fobia. Esta dualidad había sido introducida en realidad por Eugen Bleuler en su libro sobre la esquizofrenia: él hablaba de síntomas fisiógenos -dependientes de la alteración neurofisiológica que subyace en el morbus dementia praecocis, es decir, en la enfermedad esquizofrenia- y síntomas psicógenos, referidos a la elaboración biográfica que el enfermo hacía como sujeto con los síntomas de la enfermedad. Esta dualidad fue visible antes en un discípulo de Bleuler, C. G. Jung, que en 1905 publicó un libro muy inteligente, Psicología de la demencia precoz, en donde ya se recoge la influencia del pensamiento psicoanalítico, sin abandonar el punto de vista clínico bleuleriano, y después en la monografía de Wyrsch, también seguidos de Bleuler, La persona del esquizofrénico.

\section{Limitaciones teóricas en la Psiquiatría de 1940}

\section{El «nacionalismo» psiquiátrico}

¿Qué inconvenientes tenía aquella psiquiatría vista desde ahora? Tenía uno, gravísimo: el nacionalismo. El patriotismo es una de las lacras, una de las enfermedades de las cuales deberíamos vacunarnos lo antes posible para no ser jamás 
ni nacionalista ni patriota. Pues bien, el nacionalismo y el patriotismo contaminaron también a la psiquiatría. La psiquiatría francesa era ignorada por los alemanes y a la inversa; los ingleses ignoraban la psiquiatría de franceses y alemanes... y así sucesivamente. Esto convirtió a la psiquiatría en el gran escándalo de la medicina del momento, porque la clasificación de los trastornos mentales era distinta no sólo según los países, sino además, dentro de cada país, según las escuelas. Es decir que la nosología de Kraepelin no era la de Bumke; ni la de Kraepelin y Bumke era la de Kleist; y lo mismo ocurría en Francia entre la de Baruk y la de Delay o Henry Ey. Esto es verdaderamente escandaloso y, por lo tanto, el que se haya superado este nacionalismo llevando las cosas a un terreno estrictamente neutral, como es el del conocimiento científico, me parece muy importante. Aunque esto tiene su faceta negativa, y, permítanme que lo diga, muy negativa, a saber: que para que haya acuerdo en la catalogación de los síntomas se ha tenido que ir a una simplificación de las descripciones de los síntomas y síndromes, simplificación que, en el plano de la conceptualización, conlleva errores gravísimos. Volveré luego sobre esta cuestión, que ahora no es el momento.

\section{El cese de la investigación biológica}

El segundo problema fue el estancamiento de la investigación biológica. Después de los descubrimientos de Gjessing y Greving, que por supuesto están muy superados y nadie se acuerda de ellos, hay un estancamiento porque no se sabe por dónde seguir. En los Archives of Neurology and Psychiatry, norteamericanos, se ven claramente los palos de ciego que se están dando en la investigación llevada a cabo por el grupo de Altschule, que era uno de los investigadores más renombrados de la psiquiatría biológica del momento. Se buscaban las alteraciones fisiológicas en órganos como el hígado, el bazo, el humor vítreo, en los sitios más inverosímiles porque había una desorientación total, y no se contaba con una hipótesis de trabajo verosímil para ir hacia un camino fecundo.

3. El «autismo» de la psiquiatría europea

También había otras cuestiones que, en cierto sentido, se reproducen actualmente. Me refiero al, por así decir, autismo de la psiquiatría europea y, concretamente, de la alemana, que se pone de manifiesto en una fuerte sordera frente a conocimientos que no siendo estrictamente psiquiátricos, pueden fecundar a la psiquiatría. De la misma manera que un topógrafo necesita saber al mismo tiempo de óptica y 
matemáticas, nosotros, los psiquiatras, tenemos que saber de algo más que de psiquiatría stricto sensu. Lo curioso es que el psiquiatra pasaba por ser de los médicos más cultos, pero yo me resisto a que esa afirmación se haga de una manera tan tajante. Hay dos disciplinas, la Sociología y la Lingüística, que han sido completamente ignoradas por la psiquiatría desde el comienzo del siglo XX, y podríamos decir que hasta ahora, salvando algunos momentos de predominio de la psiquiatría social.

\subsection{Ante la sociología}

El Suicidio de Emile Durkheim se publicó en 1899 y el libro sobre el suicidio de Gruhle en 1910. Pues bien, este último desconoce la publicación del primero. El libro de Durkheim es revolucionario en todos los órdenes de las ciencias humanas, especialmente en la Sociología. Si la investigación sobre el suicidio de Durkheim hubiera tenido una proyección en la psiquiatría de la época, ésta se hubiera enriquecido y hubiera adquirido facetas muy distintas, más amplias, más profundas. Esto no ocurría en Estados Unidos, porque al ser un país de inmigrantes, las condiciones socioculturales saltaban a la vista. En Estados Unidos se publicaron en 1902 trabajos pioneros y se advierte que, durante los dos primeros años de la llegada, la tasa de lo que hoy llamamos brote psicótico en napolitanos y sicilianos, noruegos o lituanos, etc. era un 50\% superior respecto de los que llevaban en el país 5 ó 6 años y ya estaban integrados en la sociedad norteamericana. Esto les llevó a considerar la importancia de este desajuste del medio como un factor cuando menos desencadenante.

Otro ejemplo de esta sordera mental es el hecho de que uno de los primeros trabajos de sociología realizados por un psiquiatra en Europa, anterior a la publicación de su Psicopatología, es de Karl Jaspers. Jaspers publicó su psicopatología a los 32 años, pero varios años antes estudió el incremento de la tasa de delirios de infidelidad, lo que los alemanes llamaban «celotipia», en la población de inmigrantes en Alemania. Y subraya que es precisamente en el momento en que se produce la inmersión en un ambiente nuevo, en un mundo nuevo cuyas claves no se comprenden, cuando surge esa perplejidad frente a la interpretación de la realidad que, entre otros trastornos, conlleva la celotipia.

\subsection{Ante la lingüística}

Deseo señalar otro caso muy grave de dar la espalda en psiquiatría a ciencias que pueden enriquecerla. Es el caso de la ignorancia de las investigaciones sobre el 
lenguaje. Si se piensa que los síntomas de los cuadros psiquiátricos los detectamos porque se nos describen, es decir, se nos habla, resulta incomprensible que se pudiera hacer psicopatología ignorando la obra de Ferdinand de Saussure, Lingüística general, de 1916, y las dos obras clásicas del psicólogo Karl Bühler, Teoría de la expresión y Teoría del lenguaje, que son de las décadas de los años veinte y treinta. Algunas de las desafortunadas postulaciones de Schneider acerca de la estructura de las ideas delirantes se hubieran evitado si la obra de Saussure la hubiera conocido, y me refiero tan sólo a su distinción entre significante y significado.

\subsection{Psicopatología sin psicología}

Otra fallo de la psicopatología de entonces era el hecho, escandaloso, de que se hizo al margen de la psicología. Desde el punto de vista epistemológico, esto es tan absurdo como si la fisiología patológica (lo que conocemos como patología general) que estudiamos en medicina se hiciera al margen de la fisiología. Todos sabemos que no hay posibilidad de entender la patología funcional del riñón si no conocemos la fisiología del riñón. Sin embargo, los conocimientos de psicología que tiene el psiquiatra o son nulos o se caracterizan por su pobreza... No obstante, había un acento positivo en el psiquiatra de entonces respecto del de ahora. Creo que veíamos al enfermo, sobre todo al psicótico, como Claude Bernard decía que se debía ver al enfermo, a saber, como un experimento de la naturaleza. El «caso» -en esquizofrénicos, paranoicos, en psicosis determinadas por enfermedades generales, etc.- era estudiado más pormenorizadamente.

Después de la Segunda Guerra Mundial la psiquiatría norteamericana penetró en la psiquiatría europea, lo que supuso otro rasgo característico, como es el abandono de la investigación neuropatológica, que tomaría otro carácter años después. Un gran neuropatólogo alemán que se exilió en Inglaterra, Meyer, llegó a confesarme, en su paso por Córdoba, que había vivido el drama de haber dedicado su vida a una investigación que no tenía futuro. La neuropatología pasó entonces a un segundo plano dejando su lugar a la neurofisiología aplicada a la farmacología, y por otra parte la psicopatología adquiere una impronta psicoanalítica que fue realmente una barrera, pese a la enorme difusión que tenía entonces, porque era una psicopatología desde un punto de vista escolástico, en este caso el psicoanalítico, aunque, desde mi punto de vista, la psicopatología de aquel momento y en aquellas latitudes muestra su mayor riqueza. En este sentido, y la señalo como ejemplo, la obra de Harry Stack Sullivan, psiquiatra y sobre todo psicopatólogo, es de capital importancia. Les recomiendo lean su obra si quieren enriquecerse desde todos los puntos de vista. 
Nueva psiquiatría

\section{Psicofármacos}

La nueva psiquiatría que caracteriza al psiquiatra de hoy comienza en 1952, un año trascendental: aparecen la clorpromacina y la reserpina como los primeros dos antipsicóticos, y la imipramina como el primer antidepresivo. Lo que ocurre entonces tiene su paralelo tras la aparición de la isoniacida del ácido isonicotínico, que cura la tuberculosis y que provocó la desaparición de la industria hotelera suiza basada en tuberculosos ricos del mundo entero. Como desapareció en España la denominada lucha Antituberculosa y se reutilizaron todos los sanatorios del Guadarrama. En los primeros seis meses de uso, la isoniacida acabó con la lucha antituberculosa española y con la industria hotelera montada a propósito de ella. Los que por razones de edad y de suerte hemos tenido la ocasión de estrenar determinados fármacos, como en mi generación estrenamos las sulfamidas y la penicilina, y diagnosticar una meningitis meningocócica por la mañana y curarla a las 6 u 8 horas, o una meningitis tuberculosa curarse en una semana con unas tabletas de isoniacida, no salíamos de nuestro asombro. Y en el orden psiquiátrico, observar cómo con la clorpromacina y la reserpina el enfermo con un brote psicótico iniciaba a las pocas horas su crítica de las formaciones alucinatorias y delirantes, aunque luego volvieran otra vez, si, por optimismo prematuro, interrumpíamos el tratamiento, pero le oyéramos decir «es que yo me creía que todos se referían a mí, y me creía oír voces que me decían lo que tenía que hacer», etc. y reconocer que «es que mi cabeza no andaba bien», era algo que no se había visto jamás en la historia de la psiquiatría. Publiqué dos trabajos sobre el proceso de degradación de las estructuras psicóticas con los nuevos neurolépticos en 1956.

Un psiquiatra que pertenecía a esta Asociación, Gutiérrez Higuera, uno más de entre los directores de manicomios, el de Los Prados, de Jaén, en un congreso que hubo en Madrid en 1954, a los dos años de la aparición de la clorpromacina, pronunció esta frase sin jactancia alguna: «Este año pasado se han dado más altas que ingresos en el Psiquiátrico de Jaén tras la utilización de la clorpromacina». Recuerdo que Sarró, uno de los psiquiatras más en boga y de más poder de entonces, contestó, perdonándole la vida: «Yo también receto la clorpromacina a mis pacientes; pero les digo: tómenla cuanto antes, antes de que se pase de moda».

La clorpromacina supuso realmente una ruptura en la concepción y la visión de los enfermos psicóticos. Inmediatamente, la investigación neurobiológica sustituye a la neuropatológica. Recuerdo la enorme impresión que nos produjo leer en Der Nervenartz, una revista muy prestigiosa, los primeros trabajos sobre la depleción de dopamina que tenía lugar en las mitocondrias de las neuronas una 
vez que los enfermos habían sido tratados con clorpromacina o la reserpina. Esta investigación psicofarmacológica, esta psicofarmacología que introduce una nueva línea de investigación, es la que realmente hace posible todo lo que hoy día es la asistencia psiquiátrica al enfermo mental. No sería posible si todavía estuviéramos manejando el electroshock y los comas insulínicos, estos últimos tan inútiles como peligrosos.

\section{La Psiquiatría, globalizada}

Ya señalé que otra ventaja indiscutible de la psiquiatría de hoy es el hecho de que los nacionalismos hayan sido superados. Las clasificaciones, tanto el ICD como el DSM, han introducido una unidad de criterios y, hoy día, se puede catalogar con los mismos criterios en Japón, Senegal o en Bilbao. Ésta es una enorme ventaja, porque ha acabado con los nacionalismos, pero, por contra, los psiquiatras no extraen del análisis psicopatológico, es decir, del análisis de los síntomas todo lo que sería posible, y de hecho, la psicopatología la están haciendo, y con sumo provecho, los psicólogos, sobre todo los de corte cognitivista. Un diabetólogo puede no conocer el ciclo de Krebbs sin dejar de ser un buen diabetólogo y tratar correctamente a sus pacientes. Pero un neurólogo, si al mismo tiempo que explora la sensibilidad con el alfiler, el algodón y el martillo de reflejos no sabe por dónde se transmiten esos impulsos de la sensibilidad profunda, la interoceptiva y la propioceptiva, no era un buen neurólogo (hoy la cosa es distinta, porque el diagnóstico basado en síntomas, es decir, el semiológico, ha sido sustituido por el diagnóstico basado en la imagen, lo que he llamado en otro lugar diagnóstico icónico). Pero la psiquiatría tiene que hacer algo más respecto a la valoración sintomática que no sea la mera catalogación del paciente.

La multiplicación de psicofármacos ha hecho posible que el psiquiatra se conduzca como un médico en sentido estricto. $\mathrm{Y}$ a la industria farmacéutica debemos un avance en el conocimiento de las perturbaciones metabólicas que tienen lugar en distintos sectores del cortex y hasta en distintos sectores de la neurona. Pero la industria farmacéutica tiene también su faceta negativa: no solamente cada una de estas casas farmacéuticas nos dice que su producto es el mejor, sino que cuenta con investigadores proclives a «demostrarlo», y a inventarse nuevas entidades nosológicas, como ocurrió con la fobia social y los ataques de pánico, o con conceptos epistemológicamente incorrectos, como el de la «comorbilidad», falacias que tienen como resultado un mayor consumo de fármacos ad hoc. De esa manera han surgido factores de distorsión sumamente graves sobre los cuales se debe estar advertido. 
Sin embargo, los espectaculares cambios en la fenomenología de los síntomas mentales que tiene lugar tras la administración de los neurolépticos, no ha sido suficientemente utilizada para la investigación de lo mental, es decir, de la psicopatología propiamente dicha. El desinterés del psiquiatra actual por la patología de lo mental, es decir, por la psicopatología propiamente dicha, es lamentable, tanto más cuanto que somos nosotros los que contactamos directamente con ese sujeto, experimento de la naturaleza, que es el enfermo propiamente dicho. Son los psicólogos, especialmente los del área cognitivista, los que están desarrollando esa labor y a los que habrá de agradecérselo en su momento.

En la actualidad se está intentando reiniciar una psiquiatría teórica de la mano, por ejemplo, de Ciompi, Monti, Griffith, el grupo de Fulford, etc. Pero yo no soy demasiado optimista por la forma como se está llevando a cabo.

\section{Final}

Voy a terminar. Saben que he escrito una autobiografía. Pero una autobiografía no tiene como propósito saber quién es uno, porque antes de haberla comenzado ya sabía uno cómo era y creía saber quién es. Para eso no hace falta escribir una autobiografía, que es una tarea muy pesada, sobre todo si tiene mil páginas como la mía. Pero cuando la autobiografía se inicia y se culmina, uno aparece ante sí mismo de otra manera a como se creía antes. La escritura autobiográfica ha demostrado tener, por lo menos para mí, un cometido con el que no contaba, y es el siguiente: saber de una manera, llamémosle más sólida, a juzgar por quién se es y cómo se es, qué se espera todavía de la vida que nos queda por vivir. No todas las autobiografías tienen esa virtud, aunque tengan otras más divertidas, pero las que la poseen permiten enunciar, aunque sea tardíamente, en las postrimerías de nuestra vida biológica y nuestra vida social, cuando ya es innegable que el tiempo que nos queda por vivir se está adelgazando tanto como para que ya no quepa ni un engaño más en nuestra existencia, algo tan sorprendentemente sencillo como esto: «Ya sé quién he sido y ya sé quién me queda por ser». Pues bien, en mi caso he sido psiquiatra y seré psiquiatra. Uno más. Como todos ustedes.

* Conferencia de clausura del XXIII Congreso de la AEN, Bilbao, mayo de 2006. 